\title{
Histopathological Study of Inhibitory Effects for Relapse by Relaxin after Expansion in Rat Midpalatal Suture
}

\author{
Rie Itou, ${ }^{1}$ Tadahiko Utsunomiya, ${ }^{2}$ and Masaru Yamaguchi ${ }^{3}$ \\ ${ }^{1}$ Nihon University Graduate School of Dentistry at Matsudo, Orthodontics, Matsudo, Chiba 271-8587, Japan \\ Departments of ${ }^{2}$ Oral Pathology, and ${ }^{3}$ Orthodontics, Nihon University School of Dentistry at Matsudo, Matsudo, Chiba \\ 271-8587, Japan
}

Correspondence to :

Rie Itou

E-mail : kubo.rie@ nihon-u.ac.jp

Keywords :

relaxin, inhibition relapse,

midpalatal suture

\begin{abstract}
The objectives of this study were to clarify the inhibitory effect of relaxin (RLX) on the relapse after expansion of the midpalatal suture in rats, and to assess the expressions of type I collagen and matrix metalloproteinase-1 (MMP-1) using the immunohistochemical and image analysis techniques. Twenty four Wistar male rats (age: six weeks old, body weight: about $200 \mathrm{~g}$ ) were used in this study. They were divided into the placebo injection group (control group) of 12 rats and the RLX injection group (experimental group) of 12 rats. The expansion period was set to 7 days and the amount of relapse was measured for 14 days. After expansion, immunohistochemical analyses of type I collagen and MMP-1 were performed on 1, 3, 7, 14 days. The amount of expansion was $0.37 \pm 0.05 \mathrm{~mm}$ in the experimental group and amount of expansion was $0.32 \pm 0.08 \mathrm{~mm}$ in the control group. The amount of relapse in the experimental group was inhibited about $40 \%$ than that of the control group on the 14 days. The type I collagen and MMP-1 showed positive immunoreactivity in the fibroblasts, connective tissues and osteoblasts of the midpalatal suture area. The injection of RLX increased the expression of MMP-1 and decreased that of type I collagen after the expansion of the midpalatal suture, comparing with the control group. The results suggest that RLX may reduce the relapse after expansion of the midpalatal suture through its antifibrotic properties.
\end{abstract}

\section{Introduction}

Rapid lateral expansion of the maxillary bone is applied to malocclusion accompanying a narrowed maxillary dental arch or the maxillary bone itself in order to improve occlusal function by expanding the width and diameter of the dental arch. The purpose of this technique is to open the midpalatal suture area of the maxillary bone and maintain the maxillary dental arch width increased by adding new bone to the area (1). For changes after expansion, it has been demonstrated that during tooth movement and the alveolar bone is modified at an early stage, then, the midpalatal suture is opened up to the maxillary complex resulting in a series of changes (i.e., combination movement) (2).
This technique of lateral maxillary expansion was reported by Angle (3) in 1860. Since then, the technique has been widely used for expansion of the maxillary bone in the clinical field. The drawback of this technique, however, is the relapse after removal of the appliance. The causes of relapse include delayed bone formation after dynamic orthodontic practice, reabsorption of the formed bone, the occurrence of stress-strain accumulated in the maxillary bone itself or the suture area with adjacent bone and the tensile force and occlusal force generated in the soft tissue (palatal mucosa, buccal mucosa, etc.) (4). In order to prevent relapse after rapid expansion, it is thought that the use of retainer for long term for the remodeling of bone at the midpalatal suture area 
and the loosening of fibers must be necessary.

The insulin/insulin-like growth factor (IGF) family play an important role in accelerating the remodeling of bones. IGFs also enhance the proliferation of chondrocytes and the proliferation of their collagen and proteoglycan matrix (5). Relaxin (RLX), which belongs to the insulin/IGF family, is a hormone with a molecular weight of $6,000 \mathrm{Da}$. It is mainly produced by gestation and the prostate, the ovaries and the placenta of mammals (6). RLX is produced during the gestation of many mammals and localized in various cells and tissues (6). Recent studies have reported that it not only dilates blood vessels, grows new blood vessels and heals injuries in the brain and heart, but it also has effects on the activities of other regions including ligaments, collagen and fibroblast (7-14).

One of the biological functions of RLX is to stimulate the metabolism of collagen, which is the main component in all of the organs in the body (15). RLX reduces collagen synthesis, and accelerates the production of matrix metalloproteinases (MMPs) that cause antifibrotic properties (8-14). Several studies have reported that RLX inhibits the growth of the collagen fibers and the excessive expression of collagen, which causes fibrosis $(16,17)$. Immunohistochemical studies have shown that RLX is localized not only in the ovaries but also in the skin, lungs, liver, kidneys, heart and oral cavity $(8,18)$. Yamaguchi et al (18). also confirmed RLX in the midpalatal suture. These findings imply that RLX is closely linked to collagen metabolism at the midpalatal suture area. Furthermore, Takano et al (19). reported that RLX decreased the gene expression of type I collagen and increased that of MMP-1 in stretched human periodontal ligament cells in vitro. However, very little is known about the effects of RLX on the prevention of relapse after orthodontic treatment.

The present study investigates the effect of RLX injection on the relapse after expansion of the midpalatal suture in rats, and on the expressions of type I collagen and matrix metalloproteinase-1 (MMP-1) using the immunohistochemical and image analysis techniques.

\section{Materials and Methods}

\section{Experimental animals}

Twenty four Wistar male rats with a weight of about $200 \mathrm{~g}$ at 6 weeks old (Sankyo Labo Service Co., Tokyo, Japan) were used in this experiment. Experimental animals were randomly divided into the placebo injection group (control group) of 12 rats and the RLX injection group (experimental group) of 12 rats. The period to expand the midpalatal suture area was set to 7 days to obtain a sufficient amount of expansion. The animals were reared in SPF clean cages at the Experimental Animal Center of Nihon University School of Dentistry at Matsudo. Cages, floor mats, drinking water and powder diets were all sterilized. The rats freely drank potable tap water and ate MR powder diet for (Sankyo Labo Service Co., Tokyo, Japan). All animal experiments were performed in accordance with the Ethical Guidelines for Animal Experiments of our institution (approval No. 05-0032).

\section{Expansion of midpalatal suture method and RLX injection}

Sodium pentobarbital (Somnopenty ${ }^{\circledR}$, Kyoritsu Seiyaku Co., Tokyo, Japan) was injected into the rats as interperitoneal anesthesia to eliminate pain when attaching the appliance. Round stainless steel wire with a diameter of about $0.5 \mathrm{~mm}$ was bent in a spiral manner using Tweed loop bending pliers to make metal rings with a thickness of $1.5 \mathrm{~mm}$. The metal rings were inserted and fixed between the left and right maxillary incisors to open the incisors and expand the midpalatal suture area indirectly (20) (Fig. 1). For measurement of the palate, the distance between the cervical areas on the lingual side of the maxillary bilateral first molars was measured so that the increase of the dental arch width would not be affected by the opening of the midpalatal suture area and the increased amount of tipping movement of the teeth (Fig. 2). The distance between the cervical areas on the lingual side of the maxillary bilateral first molars was measured 7 days after attaching the appliance.

Immediately after the removal of appliance, a 
placebo (20 mmol/1 sodium acetate) or recombinant human RLX-2 $(80 \mu \mathrm{g} / \mathrm{ml}$ in PBS, Peprotech, Paris, France) was injected by syringe (Hamilton microsyringe, Hamilton Co., Nevada, USA) (21). Total $3.2 \mu \mathrm{g} /$ $40 \mu 1$ of RLX was injected to the midpalatal suture

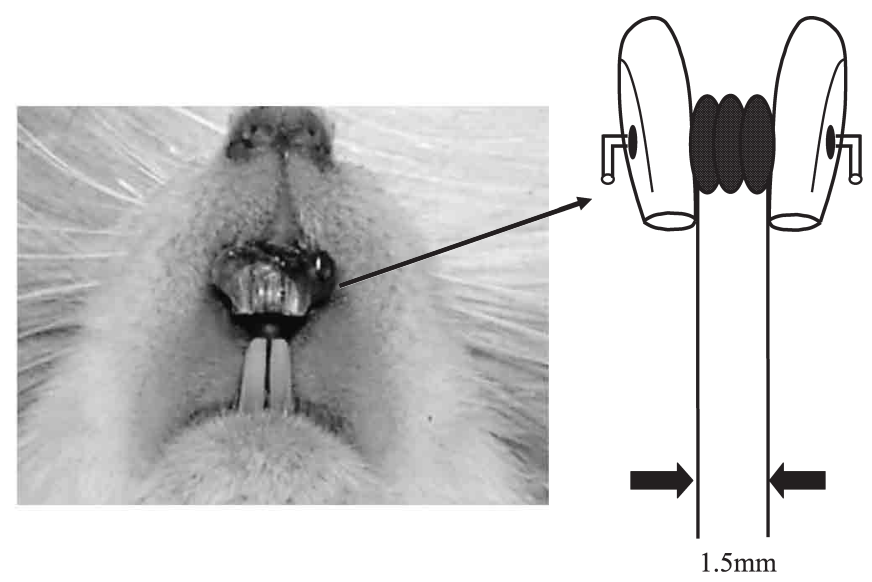

Fig. 1. Midpalatal suture expansion appliance and attaching in the rat oral cavity
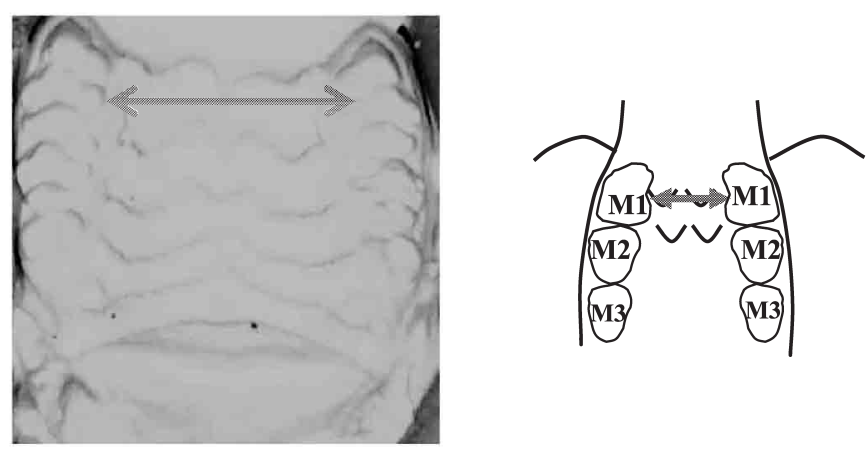

Fig. 2. Measurement of the distance between the cervical areas on lingual side of the maxillary bilateral first molars

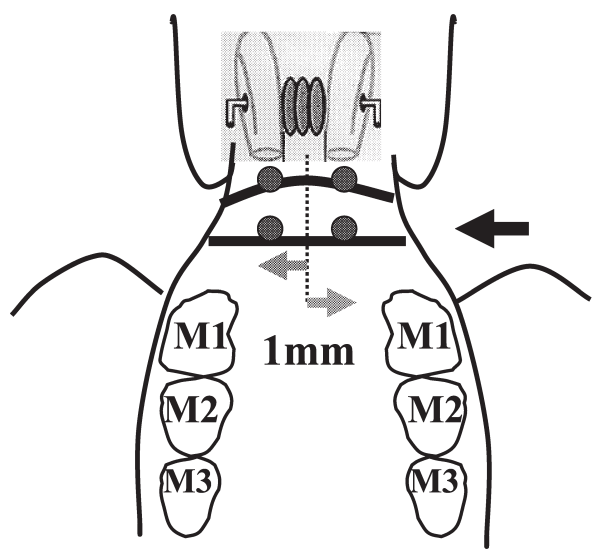

Fig. 3. Injection points of RLX or placebo area. Each $10 \mu 1$ of RLX was applied to four points. The injection points of RLX $(10 \mu 1)$ were showed in Fig. 3.

Time-dependent changes and histological changes in amount of relapse were observed 1, 3, 7 and 14 days after the removal of appliance.

Measurement of midpalatal suture expansion and amount of relapse

In order to measure the midpalatal suture expansion and amount of relapse, oral cavity impressions of the rats were obtained (three impressions per rat) by using dental rubber elastic material (DentSilicone $-\mathrm{V}$, Shofu Inc. Kyoto, Japan) before attaching the appliance, after completing expansion (7 days after attachment of appliance) and before euthanasia (1, 3, 7 and 14 days after completing expansion), for preparation plaster models were prepared. Three points, including the left and right palatal interdental papillae between the first and second molars and the palatal interdental papilla between the left second and third molars of the plaster figure were set as the reference plane.

The distance between the left and right lingual side cervical areas of the first maxillary molars was measured with a scan pitch of $0.05 \mathrm{~mm}$ by using a contact type three-dimensional scanner, PICZA (3DPicza, Roland DG Corporation, Shizuoka, Japan) and three-dimensional form analysis application software (3D-RUGLE3, Medic Engineering Co., Kyoto, Japan).

\section{Preparation of the histopathological samples}

After anesthetizing the rats, about $250 \mathrm{ml}$ of normal saline was injected into the left ventricle for avascularization. Perfusion fixation was performed with about $250 \mathrm{ml}$ of $10 \%$ neutral formalin fluid. The maxillary palate area was removed and decalcified with 10\% EDTA solution (pH 7.4) for about 14 days. According to the routine method, paraffin-embedded blocks were prepared and horizontally cut into slices with a thickness of $4 \mu \mathrm{m}$ to obtain tissue sections. 


\section{Histopathological and immunohistochemical staining technique}

Hematoxylin-eosin $(\mathrm{H}-\mathrm{E})$ staining was performed on tissue sections as the routine staining technique. Histo-fine Simple Stain Rat MAX-PO(R) (Nichirei Co., Tokyo, Japan) was used for immunohistochemical staining. Each section was deparaffinized and hydration. Then, the sections were rinsed with PBS and reacted with 3\% hydrogen peroxide methanol liquid for 15 minutes at room temperature (to block the endogenetic peroxidase reaction). The primary antibodies used included rabbit anti-human MMP-1 polyclonal antibody (Thermo, California, USA, already diluted and prepared), and rabbit anti -rat type I collagen polyclonal antibody (Calbiochem, San Diego, California, USA, dilution ratio 1 : 100). The sections were reacted over night at $4{ }^{\circ} \mathrm{C}$.

After reaction with the primary antibody, the sections were reacted with histo-fine simple stain rat MAX-PO(R) reagent (Dako Cytomation, Kyoto, Japan) at room temperature for 30 minutes. Finally, all sections were counterstained with Mayer's hematoxylin solution (nuclear staining) and were observed with a light microscope.

Positive control tests for all antibodies were performed on submucosal fibrous tissues of rats. For negative controls, the primary antibodies were replaced with rabbit serum for $\mathrm{MMP}^{-1}$ and type I collagen, then, no staining was obtained.

The center half of the suture area from the inferior division of the incisors to the front edge of the frontal palatine foramen was observed (22) (Fig. 4).

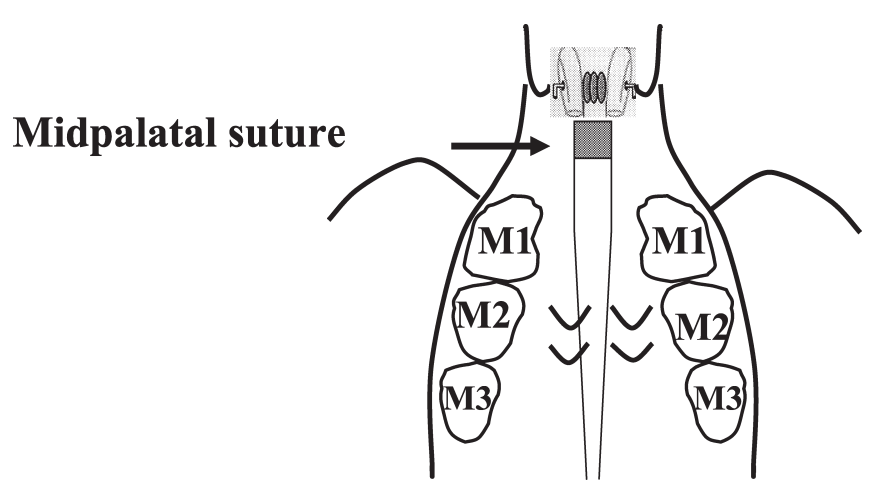

Fig. 4. Observation area of histopathology and immunohistochemistry
Measurement of stained area of type I collagen and $M M P-1$

The densely-stained area was measured using Image-Pro Plus software (version 5.0 : Media Cybernetics, Maryland, USA) (23) and the area ratio (\%) was measured by the following equation:

Area ratio $(\%)=($ Positive area of type I collagen or MMP-1 midpalatal suture area/Total area of midpalatal suture area) $\times 100$

\section{Statistical analysis}

In the statistical significance test, $t$-test were performed on the amount of relapse, type I collagen and MMP-1. The values of $\left({ }^{*}\right) \mathrm{p}<0.05$ and $\left({ }^{* *}\right) \mathrm{p}<0.01$ were considered to indicate a significant difference.

\section{Results}

Measurement of body weight

Body weight of both experimental and control groups were measured every day during the experimental period. The body weight of experimental and control groups before attaching the appliance were $197 \pm 10.6 \mathrm{~g}$ and $183 \pm 16.1 \mathrm{~g}$, respectively.

Furthermore both groups showed almost a linear increase and there was no significant difference between experimental and control groups, although slight reduction $(<5.0 \mathrm{~g})$ was observed in both groups after attaching and removing the expansion appliance.

The attachment and removal of the appliance did not affect their growth (Fig. 5).

\section{Measurement of expansion and amount of relapse}

a. Experimental group

The amount of expansion was $0.37 \pm 0.05 \mathrm{~mm}$ in the experimental groups. Fig. 6 shows the results of relapse after the expansion. In the experimental group, the 1 day (after removal appliance) group showed $0.08 \pm 0.02 \mathrm{~mm}$, the 3 day group showed $0.12 \pm$ $0.01 \mathrm{~mm}$, the 7 day group showed $0.12 \pm 0.01 \mathrm{~mm}$ and the 14 day group showed $0.12 \pm 0.04 \mathrm{~mm}$.

b. Control group

The amount of expansion was $0.32 \pm 0.08 \mathrm{~mm}$ in the control groups. Fig. 6 shows the results of relapse after the expansion. In the control group, the 1 day 


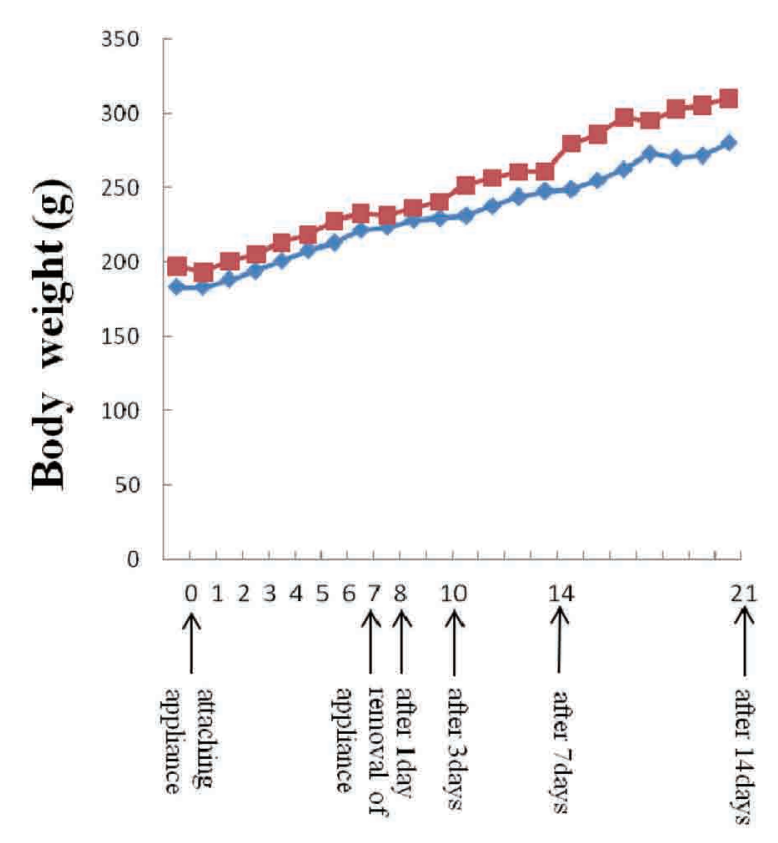

Fig. 5. Body weight

$\vartheta$ : Injection of placebo $\mathbf{a}$ : Injection of RLX

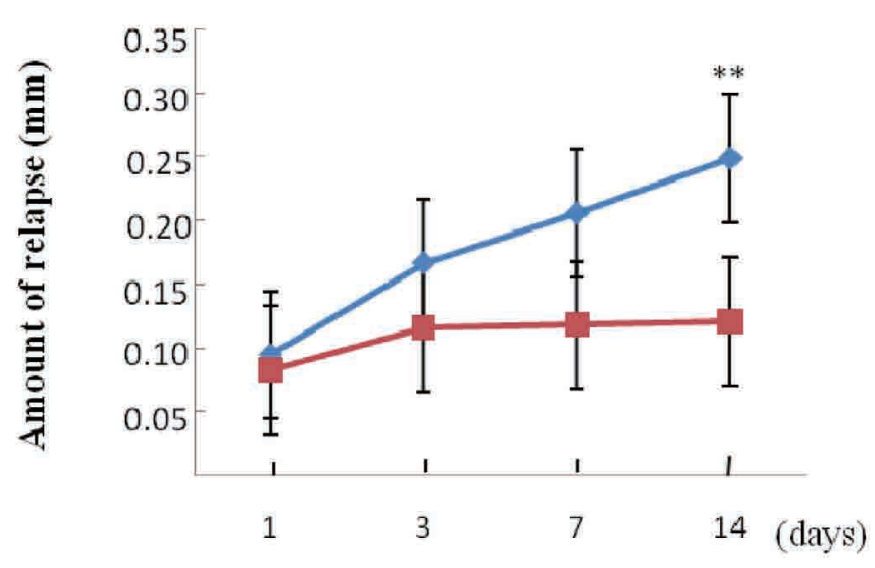

Fig. 6. Amount of relapse after the removal of appliance by RLX injection $(* * \mathrm{p}<0.01)(t$-test $)$

$\forall$ : Injection of placebo $\Xi$ : Injection of RLX

group showed $0.10 \pm 0.08 \mathrm{~mm}$, the 3 day group showed $0.17 \pm 0.06 \mathrm{~mm}$, the 7 day group showed $0.21 \pm 0.05 \mathrm{~mm}$ and the 14 day group showed $0.25 \pm 0.03 \mathrm{~mm}$.

In comparison between control and experimental groups, all the amount of the experimental groups showed a lower relapse amount than those of that control groups. A significant difference was observed during the entire period $\left({ }^{*} \mathrm{p}<0.01\right)$. The 3 day group in both the experimental group and the control group showed the largest relapse amount compared with the other observation periods.
Histopathological and immunohistochemical findings

1) Histopathological findings

a. Experimental group (RLX injection group)

The histopathological findings of the midpalatal suture area in the experimental group were shown in Fig. 7-A a-d. The connective tissues were accompanied by fibroblasts in the 1, 3, 7 and 14 day groups. The appearance of the fibroblasts in 1 and 14 day groups tended to be remarkable with that in 3 and 7 day groups. The periphery at the bone was curved irregularly in 1, 3 and 7 day groups, indicating additional bone formation (Fig. 7-A a-d).

b. Control group (placebo injection group)

The histopathological findings of the midpalatal suture area in the control group were shown in Fig. 7-A e-h. The appearance of fibroblasts in 1 day group was relatively more remarkable than that in 3 , 7 and 14 day groups. Although relatively irregular curve was also observed at the periphery of the bone in 1, 3 and 7 day groups, the findings were not so remarkable and slight when compared with experimental group (Fig. 7-A).

2) Immunohistochemical findings

(1) Type I collagen

a. Experimental group

Positive immunoreactivity was observed in the fibroblasts, connective tissues and osteoblasts (Fig. 8 $-\mathrm{A}$ a-d). The positive cell rates were $36.7 \%$ in 1 day group, $29.2 \%$ in 3 day group, $20.5 \%$ in 7 day group and $15.9 \%$ in 14 day group (Fig. $8-\mathrm{B}$ ). The positive area was significantly reduced in 3 and 7 day groups compared with the 1 day group.

b. Control group

Positive immunoreactivity was found in the fibroblasts, connective tissues and osteoblasts (Fig. 8- A e $^{-}$ h). The positive cell rates were $38.9 \%$ in 1 day group, $35.9 \%$ in 3 day group, $30.6 \%$ in 7 day group and $29.3 \%$ in 14 day group (Fig. 8-B). The positive area was reduced in the 3 and 7 day groups, however, the rate in 14 day group showed the same value as that in 7 day group (Fig. 8-B).

The positive cell rates of the experimental group were significantly decreased when compared with control group in 3, 7 and 14 days (Fig. 8-B). 
Int J Oral-Med Sci 7(3):166-175, 2009

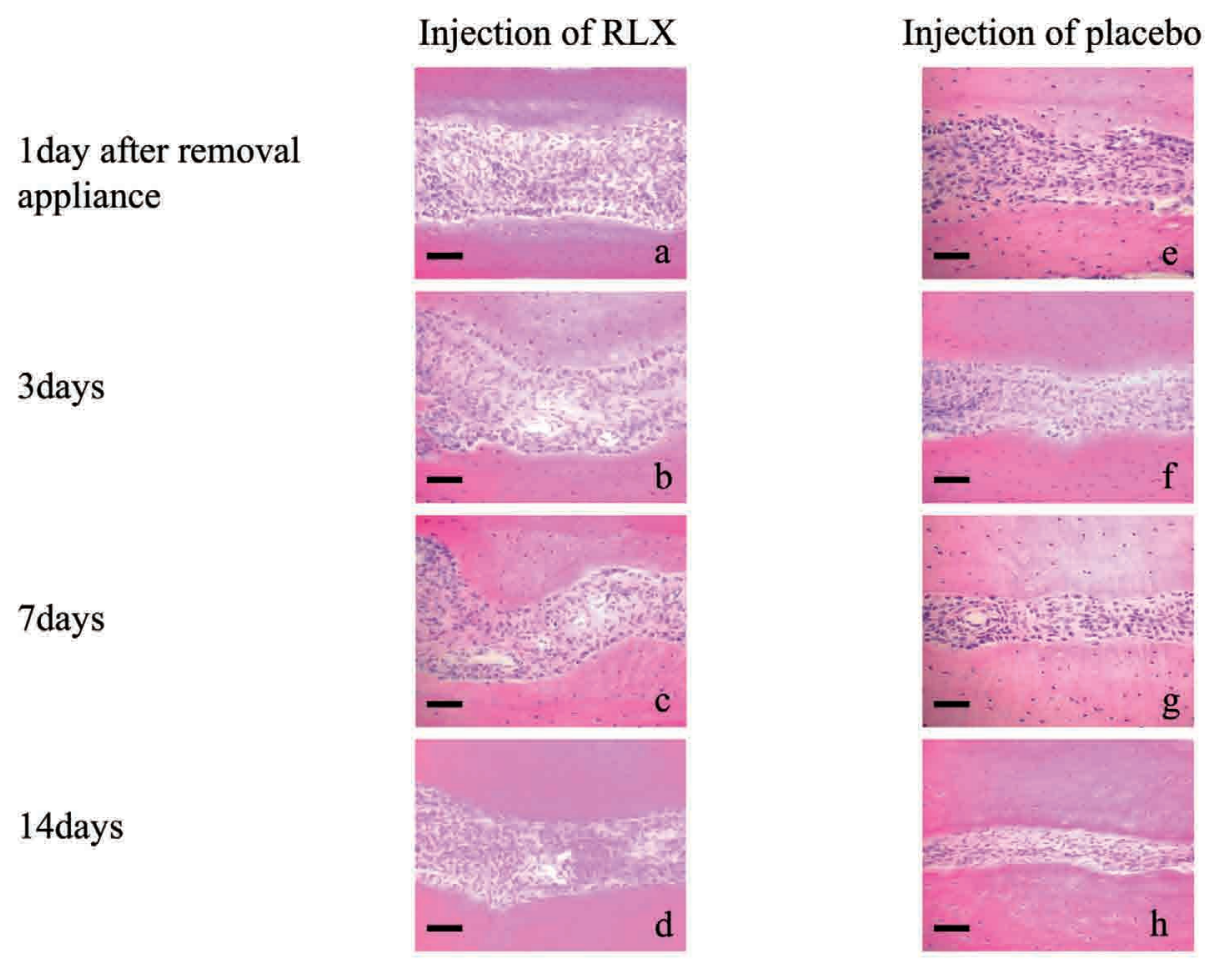

Fig. 7. Histopathological findings $(\mathrm{H}-\mathrm{E})$ in rat expanded midpalatal suture by RLX injection (bar: $50 \mu \mathrm{m}$ ) a-d: control group $\mathrm{e}^{-\mathrm{h}}$ : experimental group

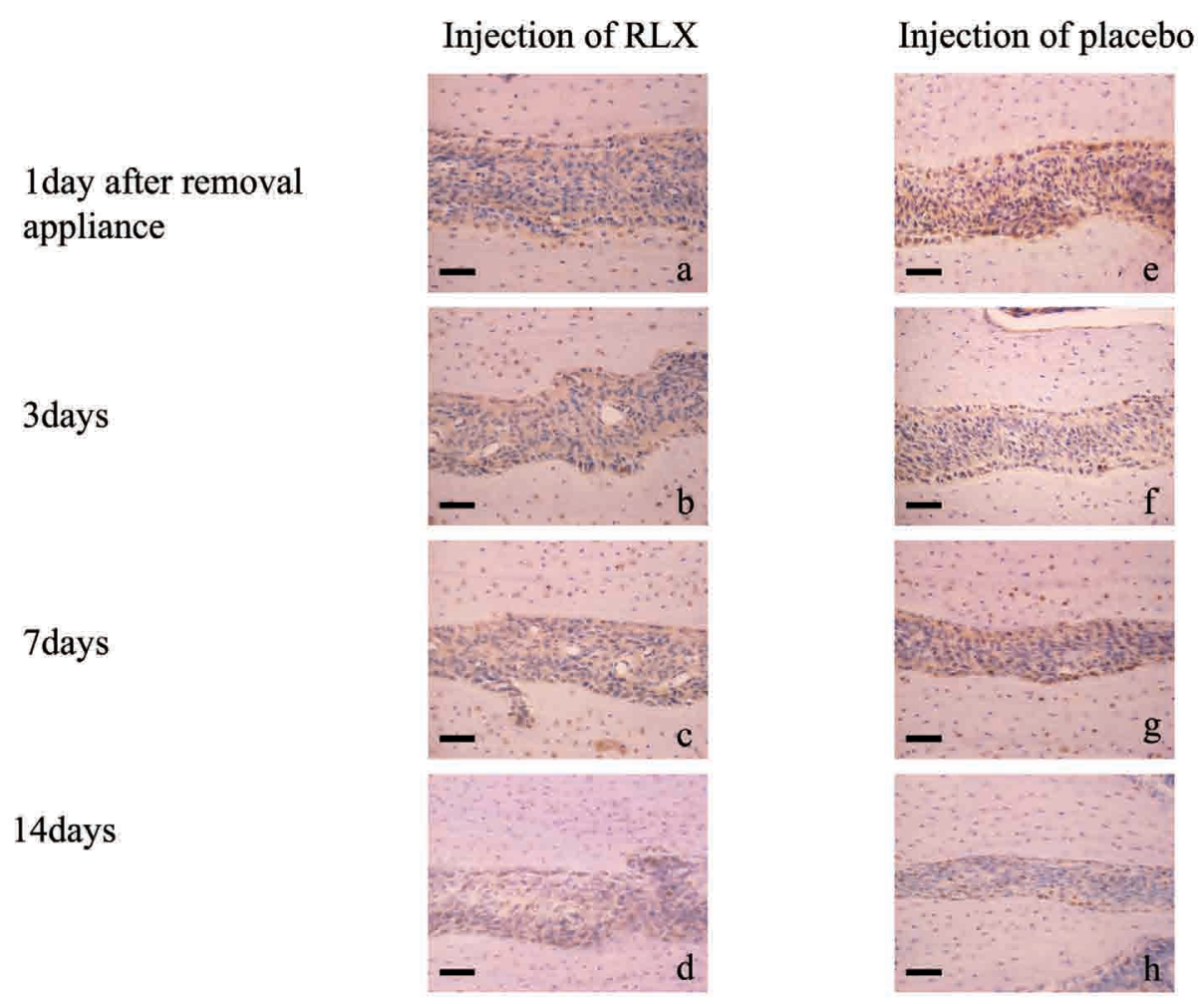

Fig. 8-A. Immunohistochemical localization of type I collagen in rat expanded midpalatal suture by RLX injection (bar: $50 \mu \mathrm{m}$ ) $a^{-} d$ : control group $e^{-h}$ : experimental group 


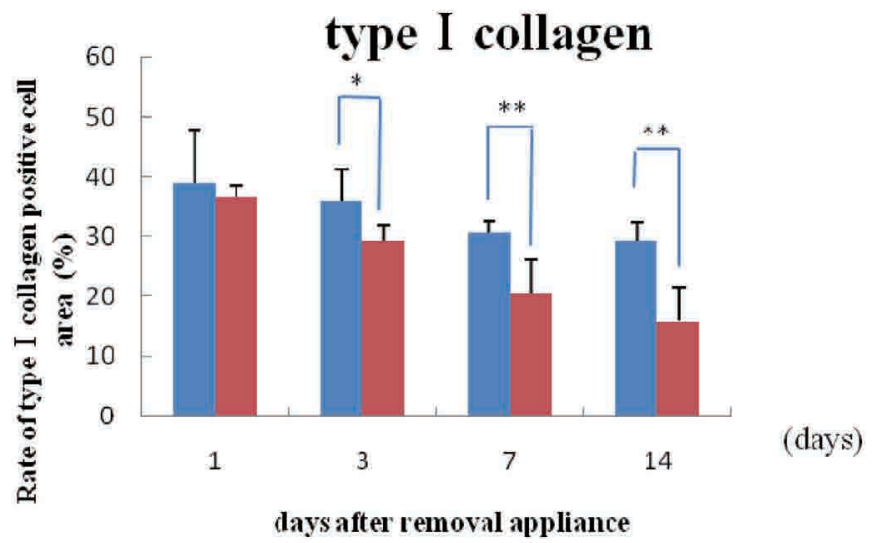

Fig. 8-B. Rate of type I collagen positive cell area after the removal of appliance by RLX injection (\%) $\left({ }^{*} \mathrm{p}<0.05,{ }^{*} \mathrm{p}<\right.$ $0.01)(t$-test)

$\underline{-}$ : Injection of placebo $=$ : Injection of RLX

\section{(2) MMP-1}

\section{a. Experimental group}

Positive immunoreactivity was identified in the fibroblasts, connective tissues and osteoblasts (Fig. 9 -A $\mathrm{a}-\mathrm{d}$ ). The area of MMP-1 positive cells was $34.2 \%, 33.9 \%$ and $33.7 \%$ in 1,3 and 7 days, respectively, indicating that the positive cell rate was stable in the experimental group (Fig. 9-B). b. Control group

Positive immunoreactivity was observed in the fibroblasts, connective tissues and osteoblasts (Fig. 9 -A e-h). The positive cell rates were $31.5 \%$ in 1 day group, $27.3 \%$ in 3 day group, $23.5 \%$ in 7 day group and $21.4 \%$ in 14 day group (Fig. $9-\mathrm{B}$ ). The positive cell rates were reduced in 3, 7 and 14 days (Fig. 9-B).

The positive cell rates of the experimental group were significantly increased when compared with control group in 7 and 14 days (Fig. 9-B).

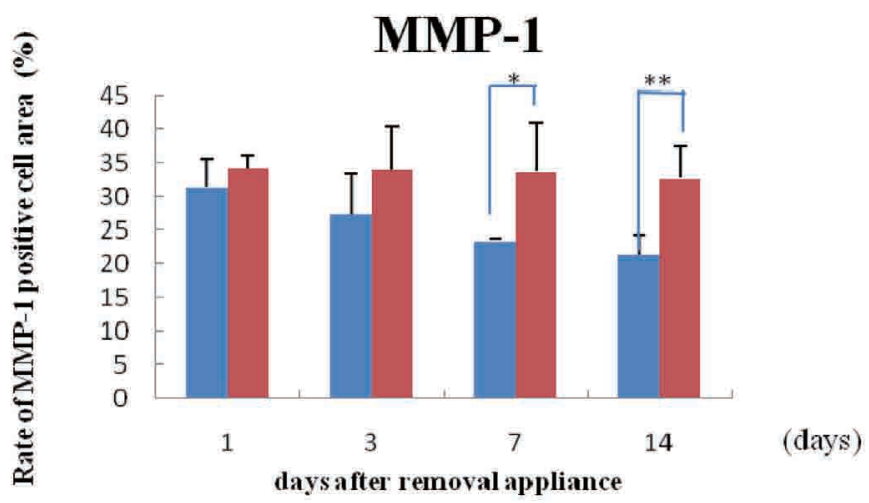

Fig. 9-B. Rate of MMP-1 positive cell area after the removal of appliance by RLX injection $(\%)\left({ }^{*} \mathrm{p}<0.05,{ }^{* *} \mathrm{p}<0.01\right)(t$-test) $=$ : Injection of placebo $=$ : Injection of RLX

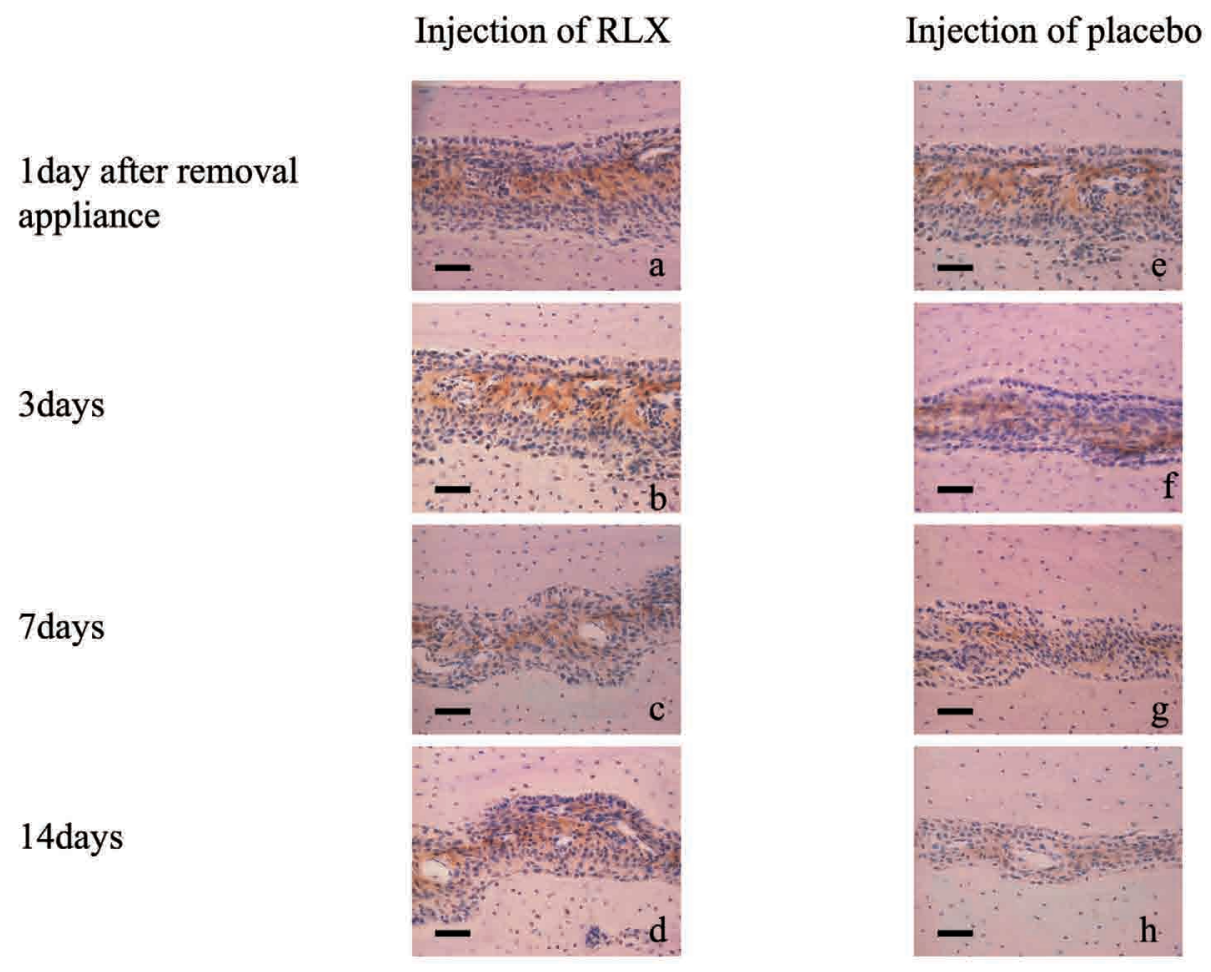

Fig. 9-A. Immunohistochemical localization of MMP-1 in rat expanded midpalatal suture by RLX injection (bar : $50 \mu \mathrm{m}$ ) $\mathrm{a}^{-} \mathrm{d}$ : control group $\mathrm{e}^{-\mathrm{h}}$ : experimental group 


\section{Discussion}

In this study we assessed the efficacy of RLX on the relapse of rapid lateral expansion of maxillary the bone.

A reduction in body weight was observed temporarily after attaching and removing the expansion appliance. The reduction in body weight may be caused by an eating disorder as a result of the changes in the oral environment caused by the operation. This reduction was slight, however, and the rats gained weight and showed growth. This suggests that the attachment of the expansion appliance had no systemic side effects (Fig. 5).

In this study, the control group showed a relatively large relapse amount of $0.10 \pm 0.06 \mathrm{~mm}$ after expansion in the 1 day group after removing the appliance. The 3 day group showed the maximum value of $0.17 \pm 0.06 \mathrm{~mm}$. Thereafter the 7 and 14 day groups, showed an increased relapse amount. Inoue et al (1). reported that the amount of relapse measurement became largest 4 days after removing the appliance in an experiment performed in the same method as in this experiment. This literature supported the results in this study. Meanwhile, the experimental group showed the maximum relapse amount of $0.12 \pm 0.01$ $\mathrm{mm}$ on the third day throughout the course of the experiment. The amount of relapse did not change throughout the experiment term (Fig. 6). Therefore, RLX may inhibit the relapse after the expansion of rat midpalatal suture.

Immunohistochemical staining showed that the expression of type I collagen (24) was significantly reduce in the 3,7 and 14 day in the experimental group after expansion (Fig. 8).

In the control group, the expression of type I collagen was not almost changed through the experimental term (Fig. 8-B). RLX is utilized to evaluate the antifibrotic properties of connective tissue in many in vivo and in vitro studies (25). Furthermore, it was demonstrated that RLX suppressed the fibrogenesis related to fibrosis and excessive collagen expression by enhancing the secretion activity of MMP (17, 26-28).

Samuel et al (15). reported that RLX injection elevates collagen metabolism by antifibrotic properties in vivo. Samuel et al. also reported that RLX suppresses collagen accumulation (fibroblasts growth), differentiation of rat heart fibroblasts stimulated by both angiotensin II and TGF- $\beta$ in vivo (16). Further Masterson et al (17). reported that RLX suppressed the reaction of the fibroblasts in the rat kidney against stimuli and matrix remodeling in vitro. Relaxin was shown to have a role in connective tissue regulation by enhancing collagen turnover (29), and Nicozisis et al (30). reported that the hormone disorganized and loosened the arrangement of the PDL from the tooth to the bone surface, and also dissolved Sharpey fiber insertions of the PDL in vitro. Furthermore, Takano et al (31). found that relaxin decreased the release and gene expression of type I collagen, and increased the expression of MMP-1 in a dose-dependent manner. Madan et al (7). suggested that enhanced PDL fiber remodeling following RLX treatment might reduce the rate or amount of relapse associated with orthodontic treatment. Whereas, in the experimental group, the expression of MMP-1 was almost stable through the experimental term. On the other hand, in the control group, the expression of MMP-1 was significantly reduced in the 7 and 14 day (Fig. 9-B). MMP-1 seemed to be continuously released in the experimental group compared with the control group. As a result, type I collagen may be decreased and caused a reduction of relapse after removing the expansion appliance.

Finally, recent study indicated that relaxin decreased the gene expression of type I collagen and increased that of MMP-1 in stretched hPDL cells (19). Taken together, these findings and our present results suggest that this hormone may be useful to prevent orthodontic relapse following orthodontic treatment.

\section{Conclusion}

The injection of RLX increased the expression of MMP-1 and decreased that of type I collagen after the expansion of the midpalatal suture. Therefore, RLX may reduce the relapse after expansion of the 
midpalatal suture through its antifibrotic properties.

\section{Acknowledgments}

I would like to say a great thank you to Professor Kazutaka Kasai for his guidance of this article. Thank you to Professors Hirotsugu Yamamoto, Toshirou Kondoh, and Masafumi Yamamoto for sacrificing their valuable time and guidance over various discussions relating to this article. Lastly, a special thank you to the staff of department of Orthodontics, Nihon University School of Dentistry at Matsudo for all the laboratory advice and help over the stressful times.

This research was supported in part by Grants-in -Aid for Scientific Research from the Japan Society for the Promotion of Science (C: 18592252, C: 19592367).

\section{References}

1. Inoue $\mathrm{K}$, Araki M, Tanba K: Influence of the systemic administration of calcitonin on the relapse after rapid lateral expansion of the maxillary bone. J Gifu Dent Soc, 28 : 68-80, 2001.

2. Aigase S, Shibasaki Y, Fukuhara T, Tachikawa T: Experimental study on the changes of the palatal soft tissue following lateral expansion in rat. J Showa Univ Dent Soc, 8: 169-179, 1988.

3. Angle DH : Treatment of irregularity of the permanent or adult teeth. Dent Cosmos, 1: 540-544, 1860.

4. Ohtake $\mathrm{H}:$ An experimental study on the changes of the palatal tissues after rapid maxillary expansion. J Jpn Orthod Soc, 43 : 16-32, 1984.

5. Schmid C: Insulin-like growth factors. Cell Biol Int, 19: 445-457, 1995.

6. Samuel CS, Hewitson TD : Relaxin in cardiovascular and renal disease. Kidney Int, 69: 1498-1502, 2006.

7. Madan MS, Liu ZJ, Gu GM, King GJ : Effects of human relaxin on orthodontic tooth movement and periodontal ligaments in rats. Am J Orthod, 131 : 1-10, 2007.

8. Samuel CS: Relaxin. Antifibrotic properties and effects in models of disease. Clin Med Res, 4 : 241-249, 2005.

9. Lekgabe ED, Kiriazis H, Zhao C, Xu Q, Moore XL, Su $\mathrm{Y}$, Bathgate RAD, Du XJ, Samuel CS: Relaxin reverses cardiac and renal fibrosis in spontaneously hypertensive rats. Hypertension, 46 : 412-418, 2005.

10. Samuel CS, Zhao C, Bathgate RAD, Bond CP, Burton MD, Parry LJ, Summers RJ, Tang MLK, Amento EP,
Tregear GW : Relaxin deficiency in mice is associated with an age-related progression of pulmonary fibrosis. FASEB J, 17 : 121-123, 2003.

11. Garber SL, Mirochnik Y, Brecklin CS, Unemori EN, Singh AK, Slobodskoy L, Grove BH, Arruda JAL, Dunea G: Relaxin decreases renal interstitial fibrosis and slows progression of renal disease. Kidney Int, 59 : 876-882, 2001.

12. Unemori EN, Pickford LB, Salles AL, Piercy CE, Grove BH, Erikson ME, Amento EP : Relaxin induces an extracellular matrix-degrading phenotype in human lung fibroblasts in vitro and inhibits lung fibrosis in a murine model in vivo. J Clin Invest, 98 : 2739-2745, 1996.

13. Williams EJ, Benyon RC, Trim N, Hadwin R, Grove $\mathrm{BH}$, Arthur MJP, Unemori EN, Iredale JP : Relaxin inhibits effective collagen deposition by cultured hepatic stellate cells and decreases rat liver fibrosis in vivo. Gut, 49 : 577-583, 2001.

14. Unemori EN, Ament EP: Relaxin modulates synthesis and secretion of procollagenase and collagen by human dermal fibroblasts. J Biol Chem, 265 : 1068110685, 1990.

15. Samuel CS, Butkus A, Coghlan JP, Bateman JF : The effect of relaxin on collagen metabolism in the nonpregnant rat pubic symphysis. The influence of estrogen and progesterone in regulating relaxin activity. Endocrinology, 137 : 3884-3890, 1996.

16. Samuel CS, Unemori EN, Mookerjee I, Bathgate RAD, Layfield SL, Mak J, Tregear GW, Du XJ: Relaxin modulates cardiac fibroblast proliferation, differentiation, and collagen production and reverses cardiac fibrosis in vivo. Endocrinology, 145: 41254133, 2004.

17. Masterson R, Hewitson TD, Kelynack K, Martic M, Parry L, Bathgate R, Darby I, Becker G: Relaxin down-regulates renal fibroblast function and promotes matrix remodelling in vitro. Nephrol Dial Transplant, 19: 544-552, 2004.

18. Yamaguchi M, Kubo R, Utsunomiya T, Yamamoto H, Kasai K : Immunohistochemical localization of relaxin in rat periodontal ligaments, dental pulp tissue, and midpalatal suture. Int J Oral-Med Sci, 7 : 35-39, 2008.

19. Takano M, Yamaguchi M, Nakajima R, Kojima T, Fujita S, Kasai K : Effects of relaxin on collagen type I released by stretched human periodontal ligament cells, Orthod Craniofac Res, in press.

20. Kanekawa M, Shimizu N : Age-related changes on bone regeneration in midpalatal suture during maxillary expansion in the rat. Am J Orthod, 114: 646-653, 1998.

21. Santora K, Rasa C, Visco D, Steinetz BG, Bagnell CA : Antiarthritic effect of relaxin, in combination with estrogen, in rat adjuvant-induced arthritis. J Phar- 
macol Exp Ther, 322: 887-893, 2007.

22. Takahashi O, Shimizu N, Iwasawa T, Hirai G: A consideration to the experimental study on the rapid palatal expansion in the rat. Nihon Univ J Oral Sci, 15: 473-481, 1989.

23. Watanabe A, Yamaguchi M, Utsunomiya T, Yamamoto H, Kasai K : Histopathological changes in collagen and matrix metalloproteinase levels in articular condyle of experimental model rats with jaw deformity. Orthod Craniofac Res, 11: 105-118, 2008.

24. Yamaguchi K : Histological study and localization of type I , II and III collagens on experimental expansion of midpalatal suture in the rat. Jpn J Oral Biol, 40 : 249-261, 1998.

25. Samuel CS, Hewitson TD, Unemori EN, Tang ML-K : Drugs of the future. The hormone relaxin. Cell Mol Life Sci, 64 : 1539-1557, 2007.

26. Birkedal-Hansen H, Moore WGI, Bodden MK, Windsor LJ, Birkedal-Hansen B, Decarlo A, Engler JA:
Matrix metalloproteinases. Crit Rev Oral Biol Med, 4 : 197-250, 1993.

27. Knauper V, Lopez-Otin C, Smith B, Knight G, Murphy $\mathrm{G}$ : Biochemical characterization of human collagenase-3. J Biol Chem, 271 : 1544-1550, 1996.

28. Samuel CS, Hewitson TD, Zhang Y, Kelly DJ : Relaxin ameliorates fibrosis in experimental diabetic cardiomyopathy. Endocrinology, 149 : 3286-3293, 2008.

29. Ivell R, Einspanier A : Relaxin peptides are new global players. Trends Endocrinol Metab, 13 : 343-348, 2002.

30. Nicozisis JL, Nah-Cederquist HD, Tuncay OC : Relaxin affects the dentofacial sutural tissues. Clin Orthod Res, 3 : 192-201, 2000.

31. Takano M, Yamaguchi M, Nakajima R, Kojima T, Kasai K: Relaxin modulates collagen type $\mathrm{I}$ and matrix metalloproteinase- 1 expression by human periodontal ligament cells. J Oral Biosci, 50: 222-229, 2008 . 